\title{
Estudio sobre la asociación de hipertrigliceridemia con los estados hipertensivos del embarazo
}

\section{Miguel Ángel Serrano-Berrones ${ }^{1 *}$ y Sergio Baltazar Barragán-Padilla²}

${ }^{1}$ Dirección Normativa de Supervisión y Calidad. Instituto de Seguridad y Servicios Sociales de los Trabajadores del Estado, Ciudad de México, México; ${ }^{2}$ Dirección General. Dirección Normativa de Supervisión y Calidad. Instituto de Seguridad y Servicios Sociales de los Trabajadores del Estado, Ciudad de México, México

\section{Resumen}

Antecedentes: La preeclampsia se presenta en el $8 \%$ de los embarazos y genera el $25 \%$ de las muertes perinatales. Aunque su etiología es multifactorial, algunas alteraciones metabólicas se asocian a la disfunción endotelial presente en la enfermedad, y su estudio podría identificar marcadores tempranos de daño. Objetivo: Determinar la relación existente entre concentración plasmática de triglicéridos en las pacientes embarazadas con enfermedad hipertensiva inducida por el embarazo. Método: Estudio prospectivo que incluyó 147 pacientes gestantes sanas y 120 mujeres con enfermedad hipertensiva inducida por el embarazo y se determinaron colesterol total, lipoproteínas de baja densidad (LDL), lipoproteínas de alta densidad (HDL), hemoglobina glucosilada $(\mathrm{HbA1c})$, biometría hemática $(B H)$, química sanguínea $(Q S)$, electrolitos séricos, nitritos séricos y pruebas de función hepática. Resultados: Se encontró una diferencia significativa en la concentración de triglicéridos entre las pacientes normotensas y con preeclampsia (261.22 \pm 80.27 vs. $361.46 \pm 135.17 \mathrm{mg} / \mathrm{dl} ; p<0.0008)$. Además, se observó una menor concentración sérica de nitritos en las pacientes con preeclampsia, que contribuye a explicar la vasoconstricción. Conclusiones: Se encontró una asociación entre la hipertrigliceridemia y la presencia de DM II con el desarrollo de la enfermedad hipertensiva inducida por el embarazo.

PALABRAS CLAVE: Hipertrigliceridemia. Preeclampsia. Embarazo.

\begin{abstract}
Background: Preeclampsia occurs in $8 \%$ of pregnancies and generates $25 \%$ of perinatal deaths. Although its etiology is multifactorial, some metabolic alterations are associated with the endothelial dysfunction present in the disease, and its study could identify early markers of damage. Objective: To determine the relationship between plasma concentration of triglycerides in pregnant patients with hypertensive disease induced by pregnancy. Methods: Prospective study that included 147 healthy pregnant women and 120 women with hypertensive disease induced by pregnancy. Total cholesterol, low-density lipoprotein, high-density lipoprotein, Hemoglobin A1c, triglycerides, BH, QS, serum electrolytes, serum nitrites and liver function tests were determined. Results: A significant difference was found in the concentration of triglycerides between normotensive and patients with preeclampsia (261.22 \pm 80.27 vs. $361.46 \pm 135.17 \mathrm{mg} / \mathrm{dl}, p<0.0008)$. In addition, a lower serum concentration of nitrites was observed in patients with preeclampsia, which helps explain vasoconstriction. Conclusions: We found an association between hypertriglyceridemia and the presence of diabetes mellitus II with the development of hypertensive disease induced by pregnancy.
\end{abstract}

KEY WORDS: Hypertriglyceridemia. Preeclampsia. Pregnancy.

Correspondencia: *Miguel Ángel Serrano-Berrones E-mail: maserrano@issste.gob.mx
Fecha de recepción: 06-03-2019

Fecha de aceptación: 19-03-2019

DOI: 10.24875/GMM.19005137
Gac Med Mex. 2019;155(Suppl 1):S27-S31 Disponible en PubMed www.gacetamedicademexico.com 


\section{Introducción}

La preeclampsia es un síndrome de etiología desconocida que se caracteriza por la presencia de hipertensión arterial y proteinuria que aparece después de la semana 20 de la gestación'. Existen cuatro trastornos hipertensivos asociados del embarazo: la hipertensión gestacional, la preeclampsia-eclampsia, la hipertensión arterial crónica y la hipertensión arterial crónica más preeclampsia sobreagregada'.

La incidencia de preeclampsia es del 6-8\% y constituye una de las principales causas mundiales de morbimortalidad maternofetal ${ }^{1}$.Cerca del $20-25 \%$ de todas las muertes perinatales son debidas a la preeclampsia, además, esta constituye una de las principales causas de restricción del crecimiento intrauterino en el recién nacido y de morbimortalidad infantil por prematurez ${ }^{1}$.

Aunque la disfunción del endotelio es la causa más reconocida en la fisiopatología de la preeclampsia, su origen aún no ha sido establecido. Se han descrito algunos factores de riesgo asociados a la preeclampsia; sobresalen la nuliparidad, la edad mayor a 35 años, la obesidad, las enfermedades crónicas como la hipertensión arterial sistémica crónica, el lupus eritematoso sistémico, la diabetes mellitus (DM), el síndrome de anticuerpos antifosfolípidos, el embarazo múltiple, la enfermedad del trofoblasto, la raza negra y concentraciones séricas elevadas de homocisteí$n^{1,2}$. Existen diversas teorías que intentan explicar la preeclampsia; entre ellas se encuentran la isquemia placentaria, la citotoxicidad mediada por lipoproteínas de muy baja densidad, factores genéticos y factores nutricionales. Entre todas ellas se puede establecer como lazo común la disfunción endotelial ${ }^{2}$.

El principal mecanismo de daño endotelial está mediado por las especies reactivas de oxígeno, o sea los radicales superóxidos, que no solamente son producidos por células fagocíticas sino también por otras poblaciones celulares. En el embarazo se sabe que la isquemia del trofoblasto genera radicales superóxido; el excedente de estos radicales produce toxicidad por conversión oxidativa de los ácidos grasos insaturados de la membrana celular, dando lugar a una peroxidación lipídica descontrolada, lo cual genera una lesión celular subletal de los trofoblastos y por ende del endotelio que lo contiene, generando además liberación de factor de necrosis tumoral alfa (TNF- $\alpha$ ). Esta última induce activación de las células endoteliales y aumento en la expresión de moléculas de adhesión de neutrófilos y plaquetas con la consiguiente liberación de enzimas y metaloproteinasas, además, el TNF- - estimula la producción del factor de crecimiento derivado de plaquetas, la actividad mitógena del endotelio y más liberación de citocinas, las cuales contribuyen a aumentar la lesión del endotelio y reduce la síntesis adecuada de óxido nítrico ${ }^{3}$.

El interés por estudiar al endotelio en las pacientes con preeclampsia-eclampsia inicia cuando deja de considerarse una estructura inerte entre la sangre y el músculo liso vascular debido al factor relajante del endotelio que es el óxido nítrico, un gas libre inorgánico, el más potente modulador de la actividad celular que se conoce; inhibe la agregación plaquetaria, relaja el músculo liso perivascular y actúa como neurotransmisor ${ }^{2,3}$.

Se ha descrito en el embarazo no complicado un aumento de los niveles plasmáticos de triacilglicerol; este incremento puede ser de hasta un 200 a 400\% en relación a niveles pregestacionales. El embarazo se puede dividir en 2 etapas: 1) anabólica debida a una hiperfagia materna y lipogénesis en el tejido adiposo predominante en los 2 primeros trimestres de la gestación, en donde se obtienen las suficientes reservas necesarias para el crecimiento fetal acelerado que ocurrirá en el último trimestre, y 2) catabólica predominante en el tercer trimestre, en donde el feto utiliza los depósitos grasos formados previamente. Esto se asocia a las mayores demandas del feto y tiene como posible consecuencia un incremento de peroxidación lipídica. Existen múltiples reportes que refieren un aumento en la concentración de los ácidos grasos libres en el suero de pacientes embarazadas con preeclampsia. La peroxidación lipídica es una reacción en cadena iniciada por el peroxinitrito, que mediante la eliminación de un átomo de hidrógeno de los ácidos grasos poliinsaturados lleva a la formación de hiperóxidos y provoca la reacción de radicales libres, causando daños al endotelio 4 .

La producción de radicales libres también genera una deficiencia de óxido nítrico por destrucción directa de este, así como por una alteración en los mecanismos de regulación de su producción a nivel endotelial $^{5}$.

La medición de la producción de óxido nítrico in vivo se realiza por medio de sus metabolitos, los nitritos y nitratos en plasma, suero u orina ${ }^{6}$. Los niveles aumentados de nitritos y nitratos encontrados en las pacientes con preeclampsia se encuentran aumentados en forma directamente proporcional a la severidad de la enfermedad, lo cual podría ser utilizado como predictor de la severidad de la 
enfermedad. Existen evidencias de que en la preeclampsia existe una disfunción celular endotelial, incluida la disminución de la producción de prostaciclina (PGI2) (efecto vasodilatador), así como un aumento de los niveles plasmáticos de endotelina $1^{7}$.

Las lipoproteínas de baja densidad (LDL) III son una fracción de las LDL ricas en triacilgliceroles que no son captadas fácilmente por los tejidos periféricos, razón por la que tienen una mayor susceptibilidad a la oxidación, a ser fagocitadas por los macrófagos, promoviendo la formación de LDL pequeñas y densas tipo III: esta fracción LDL favorece la síntesis de tromboxanos y aumenta el calcio intracelular en el músculo liso de los vasos sanguíneos, contribuyendo de esta manera al vasoespasmo y la disfunción endotelial?

Con estos planteamientos y de acuerdo con la evidencia actual, se puede afirmar que la peroxidación lipídica que conduce a un estado de estrés oxidativo juega un papel importante en el desarrollo de la preeclampsia y que las alteraciones en el perfil lipídico están implicadas directamente en este proceso. Se sabe que las concentraciones plasmáticas elevadas de ácidos grasos incrementan la oxidación en las paredes de las células endoteliales lo cual induce la sobreproducción de superóxidos por la cadena de transporte de electrones mitocondrial, con la consecuente activación de vías proinflamatorias e inactivación de PGI2 y de la óxido nítrico sintasa del endotelio ${ }^{7}$, se cree que el aumento en la concentración de óxido nítrico observado en preeclampsia a partir de la l-arginina por la óxido sintasa tiene como objetivo generar vasodilatación. El aumento en su producción podría ser debido a un mecanismo compensatorio a la vasoconstricción característica en la preeclampsia ${ }^{8}$.El objetivo del presente estudio es determinar la relación existente entre concentración plasmática de triacilgliceroles en las pacientes embarazadas con enfermedad hipertensiva inducida por el embarazo.

\section{Método}

Este estudio fue realizado en el Hospital Regional Lic. Adolfo López Mateos en el Servicio de Urgencias de Tococirugía durante el periodo del 1 de enero al 31 de diciembre de 2016. Se formaron 2 grupos de pacientes para estudio, previo consentimiento informado de estas. El grupo 1 se formó con 147 pacientes embarazadas, en el segundo y tercer trimestre del embarazo, que acudieron al Servicio para su valoración (grupo control); el grupo 2 se formó con 120 pacientes con embarazo del segundo y tercer trimestre con enfermedad hipertensiva del embarazo (preeclampsia leve o severa, hipertensión arterial sistémica crónica con preeclampsia sobreagregada, hipertensión gestacional).

Se recolectaron las muestras de sangre, previo ayuno de 8 horas. Fueron centrifugadas y se mantuvieron en refrigeración hasta su análisis. A todas las muestras se les determinaron biometría hemática, química sanguínea, electrolitos séricos, tiempos de coagulación, hemoglobina glucosilada ( $\mathrm{HbA1c}$ ), nitritos, nitratos (óxido nítrico) y perfil de lípidos (triglicéridos, colesterol total con mediciones de lipoproteínas de baja densidad [LDL] y Lipoproteínas de Alta Densidad [HDL]). (TABLA 1) Todas estas determinaciones se efectuaron por métodos colorimétricos utilizando kits comerciales. También se efectuaron determinaciones de deshidrogenasa láctica, bilirrubina directa e indirecta, y transaminasa glutámico-oxalacética y glutámico-pirúvica, así como examen general de orina para determinación de proteinuria, a las 20 pacientes que presentaron hipertensión. La estimación de producción de óxido nítrico se realizó mediante la medición de nitritos y nitratos en plasma, mediante el reactivo de Griess.

La evaluación de los resultados se efectuó utilizando la prueba t de Student para muestras no pareadas, utilizando el SPSS ${ }^{\circledR}$ versión 18.0. Un valor de $p<0.05$ fue considerado como estadísticamente significativo.

\section{Resultados}

La comparación de los parámetros basales no mostró diferencias entre grupos. Se observó una distribución por edades de ambos grupos (28.41 \pm 6.72 años en el grupo control y $32.09 \pm 5.78$ en el grupo en estudio). Se encontraron los valores séricos de colesterol $(232.78 \pm 63.15$ para el grupo control y $222.03 \pm$ 62.11 para el grupo en estudio), no presentó significancia estadística $(p>0.75)$. Esta observación se hizo extensiva tanto para los valores de las lipoproteínas de alta densidad $(45.10 \pm 27.33$ en el grupo control y $41.69 \pm 23.03$ en el grupo en estudio; $p>0.79$ ) como en las LDL (133.92 \pm 58.11 para el grupo control y $140.66 \pm 63.12$ para el grupo en estudio $(p>0.78)$.

Un resultado realmente interesante fue el obtenido con las concentraciones plasmáticas de triglicéridos. El grupo control mostró un valor promedio de 261.22 \pm 80.27 vs. $361.46 \pm 135.17 \mathrm{mg} / \mathrm{dl}$ en el grupo en estudio. La diferencia entre ambos alcanzó un valor de $p<0.0008$. En un intento por tratar de determinar si el estadio de la enfermedad hipertensiva se correlacionaba con la concentración de triglicéridos se hizo 
una evaluación bajo estos parámetros y observamos que no hubo diferencias estadísticas entre los valores de triglicéridos y la severidad de la enfermedad (403 \pm 101 en las pacientes con preeclampsia leve y 344.4 \pm 169 en las pacientes con preeclampsia severa). Los niveles de triglicéridos en aquellas pacientes con hipertensión crónica que no desarrollaron preeclampsia fueron semejantes en comparación con el grupo control $(232.60 \pm 57.13$ vs. $261.11 \pm 80.03 ; p<0.47)$.

Posteriormente se evaluó el porcentaje de pacientes con enfermedad hipertensiva inducida del embarazo que tuvieran antecedentes familiares en primera línea de diabetes e hipertensión arterial, encontrando que el $38 \%$ de las mujeres con enfermedad hipertensiva tenían dicho historial contra solamente el $10.6 \%$ de las pacientes del grupo control $(p<0.05)$. Debido a que la elevación de triglicéridos es un componente importante en el síndrome metabólico asociado con la DM y dado que la tendencia en los valores observados en cuanto al índice de masa corporal, aunque no definitivos, sí hacen sospechar una tendencia hacia este problema, evaluamos la concentración sérica de triglicéridos y de $\mathrm{HbA1c}$ en todas las pacientes. La concentración de triglicéridos en las pacientes con preeclampsia con antecedentes de DM II fue de $361.60 \pm 89.47$ vs. 257.89 \pm 80.20 del grupo control con antecedentes de DM II $(p<0.0169)$. Se sabe que las determinaciones de glucosa sanguínea están discretamente elevadas durante el embarazo, por lo que consideramos que una descompensación a más largo plazo en el metabolismo de la glucosa lo podría expresar la HbA1c. Los resultados obtenidos mostraron que no existe tal antecedente, ya que el grupo control tuvo un valor promedio de $5.116 \pm$ 0.609 y el grupo con enfermedad hipertensiva del embarazo tuvo valores de $5.011 \pm 0.509$ ( $p>0.78$ ).

Uno de los factores más importantes relacionados con la fisiología y homeostasis de estas pacientes es el óxido nítrico. Evaluamos la concentración sérica de nitritos, como producto derivado de la acción de la sintasa del óxido nítrico, enzima responsable de la síntesis del óxido nítrico. Los resultados demostraron que las pacientes con enfermedad hipertensiva inducida del embarazo tuvieron una concentración de $7.67 \pm 5.013 \mathrm{mM}$ contra $9.81 \pm 5.264 \mathrm{mM}$. Estos valores no alcanzaron una diferencia estadísticamente significativa pero sí sugieren una tendencia hacia una menor producción de óxido nítrico en las pacientes con preeclampsia, que bien puede explicar la hipertensión en estas pacientes. Es posible que dichos valores bajos se asocien con la presencia de antecedentes familiares de diabetes, los resultados mostraron que las pacientes con
Tabla 1. Comparación de resultados en los dos grupos por resultados de laboratorio

\begin{tabular}{|l|c|c|}
\hline Parámetro & $\begin{array}{c}\text { Grupo control } \\
n=147\end{array}$ & $\begin{array}{c}\text { Grupo con EHIE } \\
n=120\end{array}$ \\
\hline Edad & 28.41 & 32.09 \\
\hline Colesterol total (mg/dl) & 232.78 & 222.03 \\
\hline HDL $(\mathrm{mg} / \mathrm{dl})$ & 45.10 & 41.69 \\
\hline LDL $(\mathrm{mg} / \mathrm{dl})$ & 133.92 & 140.66 \\
\hline Triglicéridos $(\mathrm{mg} / \mathrm{dl})$ & 261.22 & 361.46 \\
\hline HbA1c $(\%)$ & 5.116 & 5.011 \\
\hline Óxido nítrico $(\mathrm{mM})$ & 9.81 & 7.67 \\
\hline
\end{tabular}

HDL: lipoproteínas de alta densidad; LDL: lipoproteínas de baja densidad; HbA1c: hemoglobina glucosilada.

Tabla 2. Comparación de grupos por niveles de triglicéridos

\begin{tabular}{|l|c|c|c|}
\hline & Hipertrigliceridemia & $\begin{array}{c}\text { Triglicéridos } \\
\text { normales }\end{array}$ & $p$ \\
\hline Casos & 361.4 & 261.1 & $<0.47$ \\
\hline Controles & 261.2 & 232.6 & $<0.47$ \\
\hline
\end{tabular}

preeclampsia con antecedentes familiares de diabetes tenían una concentración promedio de nitritos de 5.64 $\mathrm{mM}$ vs. $8.48 \pm 5.45 \mathrm{mM}$ en las pacientes sin antecedentes. Estos valores claramente refuerzan la hipótesis de que en la preeclampsia la presencia de historia familiar de DM II tiene un fuerte peso específico (Tabla 1).

\section{Discusión}

Durante la última década se han detectado tres observaciones cardinales relacionadas con la patogénesis de la preeclampsia: La primera se relaciona con la diferenciación placentaria anormal, invasión y vascularización. La segunda consiste en la disfunción de las células endoteliales, lo que explica las manifestaciones vasculares del síndrome, incluido el vasoespasmo, proteinuria, edema, hipercoagulabilidad y una producción anómala de prostaglandinas. La tercera y más reciente es la asociación de obesidad materna con un metabolismo lipídico anormal ${ }^{9}$.

La determinación de triglicéridos altos como causa de preeclampsia aplica a las pacientes de población mexicana ya que, si bien las causas de obesidad materna son diferentes entre los diversos grupos étnicos, podría ser que existieran otras anomalías aún no contempladas. Los resultados demuestran que, efectivamente, los niveles de triglicéridos en las mujeres embarazadas son más elevados que en las mujeres no gestantes. Al evaluar a las mujeres gestantes se encontró que aquellas 
que presentaron enfermedad hipertensiva del embarazo tenían valores considerablemente más elevados que las que no presentaban esta patología; sin embargo, este incremento tan importante no parece tener relación con la severidad de la enfermedad ${ }^{10}$.

El resultado confirma la hipótesis que dice que el daño endotelial secundario a la obesidad puede deberse a un incremento en la concentración plasmática de triglicéridos, ya que existe una vía metabólica bien descrita que señala que el aumento en lípidos induce un aumento en la síntesis de especies reactivas de oxígeno, las cuales favorecen la lipoperoxidación de las membranas de las células endoteliales. Además, el aumento en receptores junto con la elevación de la síntesis de óxido nítrico, que deriva del daño de las células endoteliales y que está mediado por el TNF y que además se considera un mecanismo de compensación derivado del incremento en la síntesis de endotelina 1 por daño endotelial, genera un aumento en la concentración de peroxinitritos y nitratos, aumentando así la disfunción del endotelio"11.

El funcionamiento adecuado de la célula endotelial requiere como factor fundamental la presencia de glucosa como fuente energética; es bien sabido que en los pacientes diabéticos o bien con resistencia a la insulina, una de las consecuencias metabólicas observadas y que se definen con mayor claridad en el concepto "síndrome metabólico" es la acumulación en el suero de lípidos. Es también cierto que existe una fuerte predisposición familiar a desarrollar preeclampsia en mujeres cuyas madres han sufrido este padecimiento. La definición bioquímica de esta tendencia familiar aún no ha sido explicada, sin embargo cada día aparecen más estudios que muestran un polimorfismo importante en la metilenetetrahidrofolato reductasa, que es una enzima que se relaciona con hiperhomocisteinemia, la cual es un factor bien reconocido de riesgo para preeclampsia y curiosamente también para obesidad.

En la diabetes se sabe que en los estadios iniciales las determinaciones tanto de $\mathrm{HbA} 1 \mathrm{c}$ como de daño vascular aún no reflejan la severidad de la descompensación metabólica; tradicionalmente se ha considerado que los valores ligeramente por arriba de la cifra máxima de $120 \mathrm{mg} / \mathrm{dl}$ no representan ninguna alteración en el metabolismo de la glucosa, sin embargo, se sabe que estas discretas elevaciones de la concentración de glucosa inducen modificaciones en la expresión de los transportadores constitutivos e inducibles de glucosa conocidos como GLUT (glucose transporters), los cuales se encargan de transportar la glucosa al interior de la célula.
Cuando la concentración de glucosa sobrepasa una cifra de $15 \mathrm{mM}$ estos transportadores se llegan a saturar y es entonces que el exceso de glucosa tiene que ser tomado por otras vías metabólicas induciendo la formación excesiva de lípidos (antecesor inmediato del síndrome metabólico). Este aumento de lípidos tiene que ser oxidado para poder ser eliminado por el sistema fagocítico, sin embargo los fagocitos activados secretan también citocinas proinflamatorias como el TNF- $\alpha$ que a su vez desregula la función de las células endoteliales. El aumento de lípidos también dispara la cascada de lipoperoxidación, agravando así el daño al endotelio ${ }^{12}$.

\section{Conclusiones}

Las pacientes con preeclampsia presentan un aumento significativo en la concentración plasmática de triglicéridos, un índice de masa corporal elevado y se presenta con mayor frecuencia en mujeres con antecedente de DM II.

Los resultados aquí presentados nos orientan a que en un futuro la concentración de triglicéridos pueda ser considerada como un factor predictivo de preeclampsia, como lo son actualmente el hematocrito, el cloruro de sodio, el ácido úrico y la creatinina, aunque se requieren mayores estudios al respecto.

\section{Bibliografía}

1. Spracklen CN, Smith CJ, Saftlas AF, Robinson JG, Ryckman KK. Maternal hyperlipidemia and the risk of preeclampsia: a meta-analysis. Am J Epidemiol. 2014;180:346-58.

2. Mendieta-Zeron H, Huerta-Coyote O. Dyslipidemia is a persistent problem in puerperium with or without preeclampsia. Clin Exp Obstet Gynecol. 2013;40:229-32.

3. Gallos ID, Sivakumar K, Kilby MD, Coomarasamy A, Thangaratinam S, Vatish M. Pre-eclampsia is associated with, and preceded by, hypertriglyceridaemia: a meta-analysis. BJOG. 2013;120:1321-32.

4. Russi G. Severe dyslipidemia in pregnancy: The role of therapeutic apheresis. Transfus Apher Sci. 2015;53:283-7.

5. Chandi A, Sirohiwal D, Malik R. Association of early maternal hypertriglyceridemia with pregnancy-induced hypertension. Arch Gynecol Obstet. $2015 ; 292: 1135-43$.

6. Demirci O, Tugrul AS, Dolgun N, Sozen H, Eren S. Serum lipids level assessed in early pregnancy and risk of pre-eclampsia. TJ. Obstet. Gynaecol. Res. 2011;37:1427-32.

7. Lewis GF, Xiao C, Hegele RA. Hypertriglyceridemia in the genomic era: a new paradigm. Endocr Rev. 2015;36:131-47.

8. Barrett HL, Dekker Nitert M, McIntyre HD, Callaway LK. Normalizing metabolism in diabetic pregnancy: is it time to target lipids? Diabetes Care. 2014;37:1484-93.

9. Shen H, Liu X, Chen Y, HE B, Cheng W. Associations of lipid levels during gestation with hypertensive disorders of pregnancy and gestational diabetes mellitus: a prospective longitudinal cohort study BMJ Open. 2016:6:e013509.

10. Manna FN, Khanam NN, Chowdhury KA, Das SN, Kabir MA, Zubyra SJ, et al. Study on Association of Maternal Serum Triglyceride with Pre-eclampsia. Mymensingh medical journal: MMJ. 2015;24:578-84.

11. Yu H, Yang Z, Ding X, Wang $Y$, Han $Y$. Effects of serum from patients with early-onset pre-eclampsia, HELLP syndrome, and antiphospholipid syndrome on fatty acid oxidation in trophoblast cells. Archives of gynecology and obstetrics. 2015;292:559-67.

12. Simmons SC, Dorn DP, Walton CM, Williams LA, $3^{\text {rd }}$, Pham HP. Hypertriglyceridemia in pregnancy. Transfusion. 2017;57:2824-5. 